\title{
Processing of temporal information: Evidence from eye movements
}

\author{
MIKE RINCK \\ Dresden University of Technology, Dresden, Germany \\ and \\ ELENA GÁMEZ, JOSÉ M. DÍAZ, and MANUEL DE VEGA \\ Universidad de La Laguna, Tenerife, Spain
}

\begin{abstract}
In two experiments, we recorded eye movements to study how readers monitor temporal order information contained in narrative texts. Participants read short texts containing critical temporal information in the sixth sentence, which could be either consistent or inconsistent with temporal order information given in the second sentence. In Experiment 1, inconsistent sentences yielded more regressions to the second sentence and longer refixations of it. In Experiment 2, this pattern of eye movements was shown only by readers who noticed the inconsistency and were able to report it. Theoretical and methodological implications of the results for research on text comprehension are discussed.
\end{abstract}

During the comprehension of discourse, comprehenders create multilevel representations of the information conveyed (e.g., Gernsbacher, 1990; Glenberg \& Langston, 1992; Johnson-Laird, 1983; Kintsch, 1988, 1998; van Dijk \& Kintsch, 1983; Zwaan, Langston, \& Graesser, 1995; Zwaan \& Radvansky, 1998). The level of representation associated most closely with "deep" understanding is the situation model or mental model of the text. Situation models serve to integrate the information stated in a text with general information supplied by the reader's world knowledge, and thus they "represent what the text is about, not the text itself" (Glenberg, Meyer, \& Lindem, 1987, p. 70). Many different aspects of a situation are hypothesized to be represented in situation models, for instance, causal, temporal, and spatial relations as well as the protagonists' goals, beliefs, and emotions (Zwaan, 1999; Zwaan, Langston, \& Graesser, 1995; Zwaan, Magliano, \& Graesser, 1995). Early studies of situation models focused mainly on spatial relations (see Graesser, Millis, \& Zwaan, 1997; Morrow, 1994; and Zwaan \& Radvansky, 1998, for reviews). Recently, however, other aspects - such as temporal relations-have received attention as well.

Preparation of this paper was supported by grants from the German Research Foundation (DFG) and the German Academic Exchange Service (DAAD) to M.R., and by Grants HA1999-0076(Integrated Action) and PB98-0431 from the Ministerio de Educación y Cultura to M.V. We thank Romy Findeisen, Silke Gruber, Frank Leonhard, Christine Maßloch, and Annette Weber for their help in conducting the experiments. We also thank Enrique Meseguer and Moisés Betancor for their patient teaching of the eye-tracking technique. Arthur Graesser, Rolf Zwaan, and an anonymous reviewer provided helpful comments on an earlier version of this paper. Correspondence should be addressed to M. Rinck, Dresden University of Technology, General Psychology, D-01062 Dresden, Germany (e-mail: rinck@ rcs.urz.tu-dresden.de).
Given the importance of temporal information in language and everyday life, it is not surprising that time is indeed an important aspect of situation models, affecting discourse comprehension in a number of ways. For instance, comprehension is impaired if the narrated order of events does not match their chronological order, as in flashbacks. Mismatches such as these impair comprehension, leading to decreases in comprehension speed and story recall (Mandler, 1986; Ohtsuka \& Brewer, 1992). Moreover, readers seem to hold a strong iconicity assumption, expecting that events narrated in adjacent clauses are contiguous in time and following a chronological order (Zwaan, 1996). Indeed, Zwaan (1996) found that sentences that violate this assumption impair comprehension (see also Anderson, Garrod, \& Sanford, 1983; Bestgen \& Vonk, 1995; Carreiras, Carriedo, Alonso, \& Fernández, 1997; Zwaan, Madden, \& Whitten, 2000).

Zwaan and Radvansky (1998) proposed a detailed account of how readers use temporal and other information to construct situation models during text comprehension. According to this framework, temporal information might be represented in a situation model in several different ways. First, readers may explicitly represent the temporal aspects of the described situation, such as the date, the time of day, or the order of events. Alternatively, readers may use temporal information to construct a model with particular temporal implications without explicitly representing the stated temporal information. For instance, the model might include a meeting point scene showing Person A waiting for Person B, which is obviously possible only if A arrived before B. Third, readers may flexibly use both strategies, depending on their tasks and goals.

A very popular method to assess the representation of temporal as well as other information in situation models is the inconsistency paradigm. In this paradigm, par- 
ticipants read short passages that are presented sentence by sentence on the computer screen. Presentation is selfpaced; that is, participants read each sentence until they feel they have understood it. At that point, they press a button to replace the sentence by the next one. Reading time of each sentence is assumed to reflect difficulty of comprehension, and it is used as the dependent variable. In a typical inconsistency experiment, each text includes an early sentence with a critical piece of information in one of two possible versions (e.g., Person A arrives at the meeting point before or after Person B). A number of sentences later, a target sentence contains information that is consistent with one version of the earlier information and inconsistent with the other version (e.g., Person $\mathrm{A}$ is waiting for Person $\mathrm{B}$ ). Both the earlier and the later sentence are critical to the situation model developed so far. Reading time for the target sentence is measured to determine whether readers monitor the relevant type of information. If they do, reading time for the target sentence should be longer in the inconsistent version than in the consistent one because it is much more difficult, if not impossible, to incorporate the inconsistent information into the integrated situation model. This is exactly what was found for spatial information (e.g., de Vega, 1995; O'Brien \& Albrecht, 1992), emotional information (e.g., Gernsbacher, Goldsmith, \& Robertson, 1992; Haenggi, Gernsbacher, \& Bolliger, 1994), character information (Albrecht \& O'Brien, 1993; de Vega, Diaz, \& Leon, 1997), and information about the protagonist's goals (Huitema, Dopkins, Klein, \& Myers, 1993).

Rinck, Hähnel, and Becker (2001) adopted the inconsistency paradigm to examine the representation of temporal information in situation models. The participants in their experiments read short narrative texts of seven to eight sentences that contained a critical piece of temporal information mentioned in the second sentence. The sixth sentence always contained a related piece of information, that was either consistent or inconsistent with the temporal information given earlier. For instance, in a text about the meeting of the two protagonists, Mark and Claudia, the sixth sentence mentioned that Claudia was already waiting for Mark when he arrived. In the consistent version of the text, the second sentence stated that Claudia arrived at the meeting point before Mark. In contrast, in the inconsistent version, it stated that Mark arrived before Claudia. Obviously, in this case, she can hardly be waiting for him when he arrives. The critical question asked by Rinck et al. was whether readers would include temporal aspects such as these in their situation models of the narratives, remain sensitive to temporal inconsistencies stated later in the texts, and show difficulties in integrating the inconsistent information into the situation model. To answer these questions, the usual sentence-bysentence reading paradigm was employed in three experiments. As expected, reading times of the critical target sentences were reliably longer in the inconsistent version than in the consistent one, yielding medium-sized effects of the temporal relation on reading times (see Cohen, 1988, for the interpretation of effect sizes).

The inconsistency paradigm has yielded clear-cut results for reading times collected with sentence-by-sentence presentation of texts, providing evidence for the representation of temporal as well as other information in situation models. Thus, it seems safe to conclude that this paradigm is reliable as well as valid. Moreover, it may be regarded as a good simulation of situations in which situation models are constructed during comprehension of spoken language. Another advantage of the paradigm is that the effects of inconsistencies are measured "online" rather than "off-line"- that is, during the process of comprehension rather than in a later memory test. Nevertheless, a persistent problem associated with the sentence-by-sentence presentation in general-and the inconsistency paradigm in particular-is that this is certainly not the natural way to read a text. Usually, the complete text is visible and available for inspection (or at least one or two pages of a longer text), and the reader is free to look back and forth during reading. This possibility may be particularly important during the processing of inconsistencies: Upon encountering the inconsistency, the reader may want to look back to earlier parts of the text, especially to the sentence containing the conflicting piece of information, in order to resolve the inconsistency. Obviously, this is impossible with the sentence-by-sentence presentation employed heretofore. Thus, one wonders whether the inconsistency effects observed for reading times of the target sentence are an artifact of the presentation technique, and how readers would process temporal inconsistencies in a more natural reading situation.

To answer these questions, it is useful to measure eye movements during the reading of complete texts instead of recording reading times of sentences that are presented in isolation. Although the existing research on eye movements during reading has focused mainly on lower level processes involved in the comprehension of single sentences (see Rayner, 1998), it might also be extended to the study of higher level comprehension processes using longer texts. In fact, several studies following this approach have recently been reported (e.g., Blanchard \& Iran-Nejad, 1987; Kaakinen, Hyönä, \& Keenan, 2002; Lorch \& Lemberger, 2001; Myers, Cook, Kambe, Mason, \& O'Brien, 2000; Vauras, Hyönä, \& Niemi, 1992; Wiley $\&$ Rayner, 2000). In the two experiments reported here, we used the eye-tracking technique to measure comprehension during the unrestricted reading of complete texts containing consistent or inconsistent temporal order relations. Using eye tracking to study on-line text comprehension allows for the recording of a large number of different aspects of reading behavior. For the present experiments, we focused on those aspects that are most directly related to processing of temporal inconsistencies-namely, firstpass fixations, regressions, and second-pass fixations of sentences. First-pass fixations of a given sentence were defined as all fixations within the sentence during the 
first reading of it, before moving on or moving back to a different sentence. Additional fixations of the sentence that occurred later, after at least one other sentence had been fixated, were defined as second-passfixations. Eye movements were recorded as regressions if the fixation of a sentence was preceded by the fixation of a different sentence presented later in the text; that is, only backward movements between sentences were coded as regressions. Moreover, for the specific purposes of this study, only regressions were analyzed that occurred after reading of the sixth sentence because earlier regressions cannot be attributed to processing of an inconsistency contained in the sixth sentence. In general, eye movements within sentences were not relevant to the present purpose. Finally, total text fixation time was computed by summing up all fixation times registered for the text.

Several patterns of fixations and regressions can be predicted from earlier experiments that employed the sentence-by-sentence reading paradigm. First, a temporal inconsistency contained in the critical target sentencethat is, in the sixth sentence-might cause readers to fixate this sentence for a longer time due to the increase in processing difficulty. In this case, fixation times would mirror the sentence-reading time observed in earlier experiments. On the other hand, the inconsistency might cause readers to look for information that might be used to check and resolve the inconsistency. Since the complete text is available for inspection, readers are free to look for that information anywhere in the text. However, two sentences are the most promising candidates for this search: If readers try to check the text for the inconsistency, they will find the conflicting piece of temporal information in the second sentence. In this case, we should observe more regressions to the second sentence and longer second-pass fixations of it in the inconsistent condition than in the consistent condition. In trying to resolve the inconsistency, readers might also consult the seventh (final) sentence of the text, which might give an explanation for the inconsistency. In this case, inconsistencies should cause longer first-pass fixations and longer second-pass fixations of the final sentence. Finally, if readers are not sure where to find the conflicting piece of temporal information, other sentences preceding the target sentence might also receive more regressions and longer second-pass fixations during the visual search process. Experiment 1 was designed to test these hypotheses. In addition, previous research has shown that readers do not always process the inconsistency deeply enough to be able to report it immediately after reading. Nevertheless, in a previous experiment, even these readers showed an increase in reading time in the sentence-by-sentence reading paradigm (Rinck et al., 2001), although not as strongly as the readers who were able to report the inconsistency. Therefore, Experiment 2 was designed to find out how the reading behavioras indicated by eye movements - of these two groups of readers would differ.

\section{EXPERIMENT 1}

Experiment 1 was designed to study eye movements occurring during the processing of information regarding the temporal order of events. In particular, the eye movements associated with processing of inconsistent temporal information were compared with those associated with consistent information. As in earlier experiments employing a sentence-by-sentence reading paradigm (e.g., Rinck et al., 2001), participants read short texts containing a critical piece of information in the sixth sentence that was either consistent or inconsistent with temporal order information given in the second sentence. Unlike stimuli used in earlier studies, each text was presented in toto on the computer screen, and the participants were free to read the sentences in any order they wished. While they read the texts, their eye movements were automatically recorded. Several aspects of reading behavior were of particular interest: first-pass fixations and second-pass fixations of each sentence, as well as regressions to earlier parts of the text.

\section{Method}

Participants. Thirty-two students from the University of La Laguna, most of them psychology undergraduates, participated in the experiment to fulfill a curricular requirement. All participants were native speakers of Spanish with normal or corrected-to-normal visual acuity. The data of 6 participants had to be excluded from the analyses because of incomplete or inaccurate recordings. Hence, the following analyses are based on the data of 26 participants.

Materials and Procedure. A total of 8 experimental passages and 16 filler passages were presented to the participants. All passages were presented in Spanish. The Appendix contains a sample experimental passage translated into English. Experimental passages consisted of seven sentences. The first sentence introduced the protagonists and the topic. The second sentence-called the time sentence - contained the critical temporal order information and existed in two different versions. It was either consistent with temporal information later given in the sixth sentence of the passage or inconsistent with it (see Sentences $2 \mathrm{a}$ and $2 \mathrm{~b}$ of the Appendix, respectively). In the consistent version, for instance, Alex's interview is scheduled after his meeting with Sabine, so it makes perfect sense that he wants to leave for the interview when she is late for their meeting. In the inconsistent version, this information contradicts the earlier statement that his interview had happened before their meeting (see Appendix). On the surface, the difference between the two versions of the time sentence was very minor. Following the time sentence, three filler sentences were included to ensure that the temporal information was no longer active in working memory when the target sentence was presented as the sixth sentence. This sentence was identical for all participants. It contained the critical piece of temporal information that was either consistent or inconsistent with the information in the second sentence. Each experimental passage was finished with a seventh sentence.

In addition to the experimental passages, 16 filler passages of 5 to 10 sentences without any contradictions were used to distract participants' attention from the temporal aspects of the experimental passages. Two fillers were presented at the beginning of the experiment, with the rest presented in alternation with the experimental passages. After calibration of the eye tracker, participants read the 24 passages one after the other, and each one was presented in toto on the screen of a computer connected to the eye tracker. Line spacing between sentences was increased for each passage until the passage filled the screen. Each sentence started on a new 
line. Presentation of the passages was self-paced, and participants were instructed to read each passage carefully enough to answer a comprehension question following each passage. For instance, the comprehension question following the sample text given in the Appendix read "Did Alex and Sabine meet on a sunny winter day?" It took participants about $25 \mathrm{~min}$ to read the passages and answer the comprehension questions.

Apparatus. Eye movements were recorded by a Forward Technologies dual-Purkinje eye-tracking system. Registration was done monocularly from the right eye at a rate of $1000 \mathrm{~Hz}$, with a resolution of $10 \mathrm{sec}$ of arc. Participants were seated approximately $70 \mathrm{~cm}$ from the screen displaying the texts. Their head position was fixated by means of a chinrest.

Design. The experiment followed a simple design with two within-subjects factors: temporal relation (consistent, inconsistent) and sentence position (1-7). First-pass fixations of sentences, regressions to earlier sentences, and second-pass fixations of sentences were used as the main dependent variables. ${ }^{1}$ In this experiment, sentences were used as the unit of analysis. For present purposes, eye movements within sentences were ignored and the corresponding fixation times were summed up. Moreover, only eye movements from any given sentence to a different sentence presented earlier in the text were counted as regressions, if the sixth sentence had been fixated before. Each participant read four consistent and four inconsistent target sentences. Across participants, each passage occurred equally often in the consistent and the inconsistent versions to ensure full combination of conditions and materials.

\section{Results}

Before the statistical analyses, first- and second-pass fixation times were weighted on the basis of the number of syllables in the sentences. In addition, individual distributions were analyzed in order to detect outliers (fixation times $2.5 S D$ above or below the participant's mean fixation time across sentences). Those values (between $1.5 \%$ and $2 \%$ of data) were replaced by the subject's mean fixation time. For each dependent measure, temporal relation $(2) \times$ sentence position (7) ANOVAs were performed, with participants $\left(F_{1}\right)$ or stories $\left(F_{2}\right)$ as random factors. Moreover, post hoc pairwise comparisons (Tukey's HSD test) were carried out to explore differences between pairs of conditions.
First-pass fixations. The weighted mean first-pass fixation durations of each sentence contained in the experimental texts are shown in the upper part of Table 1. A highly significant effect of sentence position was observed $\left[F_{1}(6,150)=103, M S_{\mathrm{e}}=80.60, p<.001 ; F_{2}(6,42)=\right.$ $\left.18, M S_{\mathrm{e}}=182.21, p<.001\right]$, involving the well-known known decrease in fixation times associated with serial order. The interaction of temporal relation and sentence position was significant only in the by-subjects analysis $\left[F_{1}(6,150)=2.4, M S_{\mathrm{e}}=80.70, p<.05 ; F_{2}(6,42)=1.24\right.$, $M S_{\mathrm{e}}=33.21$, n.s.]. Pairwise comparisons showed no significant difference between the consistent and the inconsistent versions at any position. Thus, as expected, the two slightly different versions of the second sentence did not yield different durations. In fact, they were almost identical, indicating the equivalence of both versions. Most importantly, first-pass fixation durations of the sixth sentence (i.e., the critical target sentence) and of the seventh sentence were not affected by consistency either. Thus, these first-pass fixations did not replicate the inconsistency effect observed for target sentence reading times in earlier sentence-by-sentence reading experiments.

Regressions. The mean percentages of regressions to each of the first six sentences are given in the middle part of Table 1. The main effect of sentence position was significant $\left[F_{1}(5,125)=43.2, M S_{\mathrm{e}}=210.43, p<.0001\right.$; $\left.F_{2}(5,35)=20.9, M S_{\mathrm{e}}=282.96, p<.0001\right]$. Overall, many regressions occurred in this experiment, presumably because the participants reread many texts in order to prepare for the comprehension question following each text. In addition to this general strategic behavior, there was an interaction of temporal relation and sentence position $\left[F_{1}(5,125)=6.1, M S_{\mathrm{e}}=213.75, p<.0001 ; F_{2}(5,35)=\right.$ $\left.3.4, M S_{\mathrm{e}}=159.12, p<.02\right]$. This interaction suggests that temporal inconsistencies selectively increased regressions toward some previous sentences. The post hoc pairwise comparisons revealed that when the sixth sentence was temporally inconsistent rather than consistent,

Table 1

Fixations (Fs, in Milliseconds) per Syllable and Percentage of Regressions, with Standard Deviations, in Experiment 1

\begin{tabular}{|c|c|c|c|c|c|c|c|c|c|c|c|c|c|c|}
\hline \multirow{3}{*}{$\begin{array}{c}\text { Dependent Variable and } \\
\text { Temporal Relation }\end{array}$} & \multicolumn{14}{|c|}{ Sentence } \\
\hline & \multicolumn{2}{|c|}{1} & \multicolumn{2}{|c|}{2} & \multicolumn{2}{|c|}{3} & \multicolumn{2}{|c|}{4} & \multicolumn{2}{|c|}{5} & \multicolumn{2}{|c|}{6} & \multicolumn{2}{|c|}{7} \\
\hline & $\mathrm{F}$ & $S D$ & $\mathrm{~F}$ & $S D$ & $\mathrm{~F}$ & $S D$ & $\mathrm{~F}$ & $S D$ & $\mathrm{~F}$ & $S D$ & $\mathrm{~F}$ & $S D$ & $\mathrm{~F}$ & $S D$ \\
\hline \multicolumn{15}{|l|}{ First-Pass Fixation Durations } \\
\hline Consistent & 59 & 17 & 38 & 9 & 24 & 8 & 16 & 9 & 25 & 8 & 35 & 8 & 27 & 8 \\
\hline Inconsistent & 54 & 13 & 37 & 11 & 24 & 9 & 17 & 8 & 33 & 10 & 35 & 9 & 30 & 7 \\
\hline \multicolumn{15}{|c|}{ Percent Regressions Toward Sentence } \\
\hline Consistent & 37 & 17 & 55 & 26 & 45 & 23 & 43 & 20 & 31 & 11 & 27 & 14 & - & - \\
\hline Inconsistent & 25 & 1 & 70 & 20 & 45 & 18 & 30 & 10 & 33 & 12 & 24 & 8 & - & - \\
\hline \multicolumn{15}{|l|}{ Second-Pass Fixation Durations } \\
\hline Consistent & 9 & 10 & 14 & 8 & 5 & 3 & 4 & 2 & 7 & 5 & 4 & 3 & 9 & 4 \\
\hline Inconsistent & 5 & 4 & 19 & 15 & 6 & 4 & 3 & 1 & 9 & 7 & 4 & 4 & 11 & 7 \\
\hline
\end{tabular}

Note-A dashed line indicates that the two means separated by it differ significantly. See text for details. There are no regressions to Sentence 7 because it is the final sentence. 
participants regressed to the critical second sentence more often (Tukey HSD $>13.88, p<.05$ ), whereas the opposite was true for the first sentence (Tukey HSD $>$ $13.88, p<.05)$. In fact, after participants read the inconsistent sixth sentence, most regressions were directed toward the critical second sentence, whereas regressions were distributed more evenly after participants read the consistent sentence. Thus, temporal inconsistencies caused spontaneous regressions to the second sentence instead of strategic regressions to the first sentence.

Second-pass fixations. The usual sentence position effect was obtained $\left[F_{1}(6,150)=29.3, M S_{\mathrm{e}}=41.15, p<\right.$ $\left..001 ; F_{2}(6,42)=6.8, M S_{\mathrm{e}}=43.71, p<.001\right]$, as well as an interaction of sentence position and temporal relation $\left[F_{1}(6,150)=4.8, M S_{\mathrm{e}}=25.35, p<.003 ; F_{2}(6,42)=2.64\right.$, $\left.M S_{\mathrm{e}}=23.69, p<.003\right]$. The pattern of second-pass fixations shown in the lower part of Table 1 was very similar to that of the regressions just discussed. As expected, temporal inconsistencies contained in the sixth sentence caused longer second-pass fixations of the second sentence (Tukey HSD > 4.68, $p<.05$ ). We also observed shorter second-pass fixations of the first sentence for temporal inconsistencies. This difference, however, fell just short of statistical significance (Tukey HSD $=4.66$ ).

\section{Discussion}

To summarize, the results observed in this experiment indicate fairly clearly how the participants dealt with temporal order inconsistencies they encountered near the end of the texts. Inconsistent temporal order relations caused readers to look back to the sentence that contained the critical piece of temporal order information and to spend more time rereading this sentence. In contrast, after participants read the consistent text versions, most of their regressions served to reread the complete text: Many of them originated from the seventh sentence and were directed to the first sentence, presumably in preparation for the following comprehension question. Thus, temporal inconsistencies caused spontaneous regressions to the second sentence instead of more strategic regressions to the first sentence. Thus, Experiment 1 suggests that the comprehension of temporal information is indeed disturbed if this information is inconsistent with earlier information, presumably because the process of situation model updating is made difficult or even impossible. Regarding this conclusion, the results of Experiment 1 are in perfect accordance with those of earlier research, employing the sentence-by-sentence reading paradigm (Rinck et al., 2001). However, the first-pass fixations recorded in Experiment 1 also suggest that the increase in target sentence-reading times observed in earlier experiments may be caused by the sentence-by-sentence presentation: Instead of spending additional time with this sentence, readers in Experiment 1 looked back for information that might help them to solve the inconsistency, and they spent more time rereading that information. In the General Discussion section, we will discuss the theoretical and methodological implications of this finding.

\section{EXPERIMENT 2}

Experiment 1 indicated that readers try to deal with inconsistencies regarding the temporal order of events by regressing to earlier information and rereading. By doing so, they are able to check whether the temporal relation described in the later sentence is indeed inconsistent with the earlier one, or whether it only seems so because they misremember the earlier information. This explanation assumes that readers are aware of the inconsistency and actively try to repair it in some way. However, Rinck et al. (2001) have shown that a temporal inconsistency increases reading times of the sentence containing it, even if readers are unable to report the inconsistency immediately after reading. If one assumes that the ability to report an inconsistency is closely associated with conscious processing of it (see Schooler \& Fiore, 1997, for arguments in favor of this assumption), it appears that conscious processing is not a precondition of the inconsistency effect. However, even longer reading times are observed if readers detect the inconsistency and are able to report it. This difference between reporters and nonreporters of the inconsistency poses a problem to our explanation of the eye movements observed in Experiment 1: Strictly speaking, the explanation applies only to reporters of the inconsistency, whereas nonreporters should exhibit a different pattern of eye movements. Which pattern might that be? If regressions from the critical six th target sentence back to the second sentence are more likely for reporters, then nonreporters might show longer first-pass fixations of the sixth sentence, mirroring the reading times observed with sentence-by-sentence presentation.

Experiment 2 was designed to investigate in detail the eye movements of reporters and nonreporters during the reading of a temporal inconsistency. The experiment closely followed the procedure introduced by Rinck et al. (2001), except that the sentence-by-sentence reading paradigm was replaced by the eye-tracking paradigm used in Experiment 1. Experiment 2 also complied with the requirements described by Rinck et al.: To avoid forgetting of consciously processed information, participants have to be questioned immediately after reading the passage containing the inconsistency. This necessarily directs their attention to temporal information and inconsistencies; therefore, only a single inconsistency may be presented to each participant. In turn, this requires that all experimental variables are varied as betweensubjects factors and that a large number of participants are tested, while the number of texts employed is minimal (viz., only two). Naturally, this creates limitations regarding the ability to generalize the results to other materials. We will discuss these in the General Discussion section.

\section{Method}

Participants. A total of 102 students of the Dresden University of Technology, most of them psychology undergraduates, participated in the experiment, either to fulfill a curricular requirement or 
for a small monetary payment. All participants were native speakers of German with normal or corrected-to-normal visual acuity. Participants had to meet three criteria for their data to be included in the analyses: The recordings of their eye movements had to be complete and error free, their answers to the comprehension questions had to be correct, and they had to be classified unequivocally as either reporters or nonreporters of the inconsistency. The latter criterion was handled very strictly: Only participants who mentioned the inconsistency immediately, fully, and correctly were classified as reporters (e.g., stating "Why does he leave for the interview, if he has had it already?"). Correspondingly, only participants who, after thorough questioning, insisted that nothing had seemed strange to them were classified as nonreporters. All participants who gave answers lying somewhere in between these extremes were excluded from the analyses. Due to the strictness of the criteria, the data of only 19 reporters and 34 nonreporters were included in the analyses.

Materials and Procedure. Following a practice passage containing neither critical temporal information nor inconsistencies, only two experimental passages were presented to the participants of Experiment 2. The first one contained a consistent temporal order relation, whereas the second one contained an inconsistent one. All texts were presented in German. The Appendix shows one of the experimental texts, which was also used in Experiment 1 (in Spanish) and in Experiment 4 of Rinck et al. (2001). The procedure was very similar to Experiment 1: After calibration of the eye tracker, participants read the three passages one after the other. Each one was presented in toto on a computer screen approximately $70 \mathrm{~cm}$ away from the participant and connected to the eye tracker. Presentation of the passages was self-paced, and participants were instructed to read each passage carefully enough to answer a comprehension question following each passage. Directly after reading the third passage with the inconsistency and answering the corresponding comprehension question, all participants were asked the following questions: "In the final text, did you notice anything that seemed strange or wrong to you? If so, what?" If their responses were ambiguous, they were questioned further by the experimenter. Depending on their responses, they were divided into three participant groups: nonambiguous inconsistency reporters, nonambiguous nonreporters, and ambiguous responders. Data of only 19 indisputable reporters and 34 indisputable nonreporters with complete eye-tracking data sets were included in the analyses. It took participants about 5 min to read the passages and answer the comprehension questions.

Apparatus. Eye movements were recorded by an EyeLink eyetracking system, distributed by SensoMotoric Instruments $\mathrm{GmbH}$ (Germany). The eye tracker is an infrared video-based tracking system combined with hyperacuity image processing. Two cameras (one for each eye) are mounted on a headband, together with two infrared light-emitting diodes (LEDs) for illuminating each eye. The cameras sample pupil location and pupil size at the rate of $250 \mathrm{~Hz}$. Registration was done binocularly, although monocular registration is possible as well. The resolution of eye position is $15 \mathrm{sec}$ of arc and the spatial accuracy approximately $0.5^{\circ}$. Head position with respect to the computer screen is measured with a head-tracking camera mounted on the center of the headband. Four LEDs attached to the corners of the computer screen are viewed by the head-tracking camera while the participant is facing the screen. Possible head motions are detected as movements of the four LEDs and are compensated for automatically. The compensation is better than $1^{\circ}$ over the acceptable range of head motion. Thus, it is not necessary to use a headrest or other means to secure the participant's head.

Design. The experiment followed a $2 \times 2$ design with the quasiexperimental between-subjec ts factor participant group (nonreporters, reporters) and the within-subjects factor temporal relation (consistent, inconsistent). As before, first-pass fixations of sentences, regressions to earlier sentences, and second-pass fixations of sen- tences were used as the main dependent variables, with sentences as the unit of analysis. Fixations shorter than $50 \mathrm{msec}$ were excluded from the analyses. On the basis of the results of Experiment 1 , interest focused on comparing the nonreporters with the reporters with regard to the second and the sixth sentence of each passage; the other sentences were of minor interest. For this experiment, only by-participants analyses could be computed because only one consistent and one inconsistent experimental passage were used. The sentences of these passages were similar in length; thus, it was not necessary to compute syllable fixation times.

\section{Results}

First-pass fixations of Sentence 6. The mean firstpass fixation durations of Sentence 6 are shown in the upper part of Table 2. As in the first experiment, no effect of the inconsistency on these fixation durations was observed $\left[F(1,51)=1.28, M S_{\mathrm{e}}=2.02\right.$, n.s.]. Fixation durations of reporters and nonreporters did not differ either, and there was no interaction of participant group and inconsistency [both $F \mathrm{~s}(1,51)<1$ ]. If anything, firstpass fixations of reporters seemed a little shorter than those of nonreporters, for both the consistent and the inconsistent temporal relation. Thus, these results replicate those of Experiment 1, and they are in contrast with reading times observed in earlier sentence-by-sentence reading experiments.

All regressions to Sentence 2. The percentages of participants showing regressions to the critical second sentence are given in Table 2 . Only regressions to this sentence some time after fixation of the sixth sentence were included in this analysis. After reading the consistent temporal relation, reporters and nonreporters showed an almost identical amount of regressions [ $42 \%$ vs. 38\%; $\left.\chi^{2}(1)<1\right]$. After reading the inconsistent relation, however, reporters showed twice as many regressions as nonreporters [68\% vs. 35\%; $\left.\chi^{2}(1)=5.27, p<.05\right]$.

Direct regressions to Sentence 2. To analyze regressions to the critical second sentence in more detail, we also determined how often participants looked back to this sentence directly after reading Sentence 6 or Sentence 7 . Thus, these regressions are the very first ones that participants might make after reaching the end of the text. The percentages of these regressions are given in Table 2. We found that nonreporters made these regressions in the consistent text as often as in the inconsistent one [21\% vs. $\left.29 \% ; \chi^{2}(1)<1\right]$. As expected, reporters made them much more often in the inconsistent text than in the consistent one [ $42 \%$ vs. $16 \% ; \chi^{2}(1)=3.11, p<.05$ ].

All regressions. An alternative explanation of the results just described would state that reporters did not regress selectively to Sentence 2 . Instead, they might be more careful readers, who generally regress more often. Therefore, we also analyzed all regressions that reporters and nonreporters made after reading consistent and inconsistent temporal relations. As in Experiment 1, the percentage of participants showing regressions was quite high, yielding an average of $77 \%$, because many participants decided to reread the text or parts of it before answering the following comprehension question 
Table 2

Fixations (Fs) in Milliseconds and Percentage of Regressions, With Standard Deviations, in Experiment 2

\begin{tabular}{|c|c|c|c|c|}
\hline \multirow{3}{*}{$\begin{array}{l}\text { Dependent Variable and } \\
\text { Temporal Relation }\end{array}$} & \multicolumn{4}{|c|}{ Inconsistency Report } \\
\hline & \multicolumn{2}{|c|}{ Nonreporters } & \multicolumn{2}{|c|}{ Reporters } \\
\hline & $\mathrm{F}$ & $S D$ & $\mathrm{~F}$ & $S D$ \\
\hline \multicolumn{5}{|l|}{ First-Pass Fixation of Sentence 6} \\
\hline Consistent & 3,764 & 1,443 & 3,515 & 1,336 \\
\hline Inconsistent & 4,102 & 1,368 & 3,753 & 1,368 \\
\hline \multicolumn{5}{|l|}{ All Regressions to Sentence 2} \\
\hline Consistent & \multicolumn{2}{|c|}{38.2} & \multicolumn{2}{|c|}{42.1} \\
\hline Inconsistent & \multicolumn{2}{|c|}{35.3} & \multicolumn{2}{|c|}{68.4} \\
\hline \multicolumn{5}{|l|}{ Direct Regressions to Sentence 2} \\
\hline Consistent & \multicolumn{2}{|c|}{20.6} & \multicolumn{2}{|c|}{15.8} \\
\hline Inconsistent & \multicolumn{2}{|c|}{29.4} & \multicolumn{2}{|c|}{42.1} \\
\hline \multicolumn{5}{|l|}{ Regressions to All Sentences } \\
\hline Consistent & \multicolumn{2}{|c|}{70.6} & \multicolumn{2}{|c|}{78.9} \\
\hline Inconsistent & \multicolumn{2}{|c|}{76.5} & \multicolumn{2}{|c|}{84.2} \\
\hline \multicolumn{5}{|l|}{ Second-Pass Fixation of Sentence 2} \\
\hline Consistent & 595 & 912 & 627 & 789 \\
\hline Inconsistent & 445 & 66 & 1,248 & 1,147 \\
\hline \multicolumn{5}{|c|}{ Total Text Fixation Duration (in seconds) } \\
\hline Consistent & 18.5 & 6.54 & 15.2 & 4.56 \\
\hline Inconsistent & 25.7 & 8.04 & 22.9 & 4.90 \\
\hline
\end{tabular}

Note-A dashed line indicates that the two means separated by it differ significantly. See text for details.

(Table 2). Apart from this, however, no differences between reporters and nonreporters or between the consistent and the inconsistent relation were found $\left[\chi^{2}(1)<1\right]$. Thus, reporters did not generally regress more often than nonreporters. Rather, they exhibited more regressions aimed specifically at the critical second sentence.

Second-pass fixations of Sentence 2. The secondpass fixation durations of the second sentence resembled the pattern of regressions just described (Table 2): Reporters and nonreporters did not differ from each after reading of the consistent temporal relation $[F(1,51)<1]$. After reading the inconsistent temporal relation, however, reporters spent much more time rereading the second sentence than did nonreporters $[F(1,51)=10.35$, $\left.M S_{\mathrm{e}}=3.11, p<.01\right]$. Accordingly, the ANOVA of these second-pass fixation durations yielded a significant interaction between participant group and temporal relation $\left[F(1,51)=9.98, M S_{\mathrm{e}}=3.63, p<.01\right]$.

Total text fixations. As with the regressions, an alternative explanation also exists for the second-pass fixations just described. According to this explanation, reporters might generally exhibit longer second-pass fixations than nonreporters, rather than specifically for the second sentence. To test this explanation, the total times that reporters and nonreporters spent on the complete texts were analyzed. The bottom part of Table 2 shows these total text fixation times. They did not reveal any differences between the two participant groups, either for the consistent temporal relation or for the inconsistent one [both $F_{\mathrm{s}}(1,51)<2$, n.s.]. For both relations combined, reporters even tended to be a little faster than nonreporters $\left[F(1,51)=3.15, M S_{\mathrm{e}}=234.44, p<.10\right]$. Thus, reporters did not generally show longer secondpass fixations; they did so only for the critical second sentence. Both reporters and nonreporters, however, showed longer total text fixation times for the inconsistent text than for the consistent one, replicating the results observed by Rinck et al. (2001) for sentence-by-sentence reading times [reporters: $t(18)=8.96, S E=.86, p<.001$; nonreporters: $t(33)=8.23, S E=.88, p<.001]$.

\section{Discussion}

The results of Experiment 2 illustrate quite clearly how the reading behavior of people who are able to report an inconsistency of temporal order differs from the behavior of nonreporters. Reporters did not read more slowly or more carefully in general, as indicated by the consistent text and by the total text fixations and the overall regressions of the inconsistent text. Rather, these readers showed particularly more regressions to the critical second sentence and longer second-pass fixations of this sentence. Thus, it appears that the specific eye movements associated with processing of inconsistencies and the corresponding repair processes outlined above are limited to readers who consciously notice the inconsistency and are able to report it shortly afterward. None of this specific behavior was found for readers who are unable to report the inconsistency immediately after encountering it. Moreover, we did not find reliable evidence for the hypothesis that nonreporters might show 
longer first-pass fixations of the sixth sentence. Although their fixations of inconsistent target sentence were indeed a little longer than those of the consistent sentence, and also longer than those of the reporters reading the inconsistent sentence, none of these differences was statistically significant. Future research will have to show whether this was caused by a lack of power due to the small number of reporters or whether the weak inconsistency effect observed for nonreporters in the sentence-by-sentence paradigm does not occur in a more natural reading situation. Moreover, future research will also have to establish that the results observed here may be generalized to a larger set of stimuli.

\section{GENERAL DISCUSSION}

Taken together, the two experiments reported here yield a coherent picture of the eye movements associated with processing of inconsistencies regarding temporal order during reading of short narratives. Moreover, the high concordance of the Spanish Experiment 1 and the German Experiment 2 indicates that the observed effects may be generalized across languages. Moreover, there is no reason to assume that the results reported here would not generalize to other aspects of situation models, such as the emotional, causal, spatial, or goal-related information investigated earlier with the sentence-by-sentence reading paradigm. The observed results also allow to evaluate the predictions regarding eye movement patterns that we outlined above. First, a temporal inconsistency contained in a later sentence of the text does not seem to cause readers to fixate this sentence for a longer time due to the increase in processing difficulty. Thus, the first-pass fixations and second-pass fixations of the critical target sentence observed here did not mirror the sentence-reading times observed in earlier experiments. Rather, the inconsistency caused readers to look for information that might be used to check and resolve the inconsistency. They did so in a fairly straightforward fashion: Most of their regressions were aimed at the critical second sentence, where they found the conflicting piece of temporal order information. Thus, we observed more regressions to the second sentence and longer secondpass fixations of this sentence in the inconsistent temporal relation compared with the consistent one. Longer second-pass fixations were also observed for the final sentence of the text, indicating that readers also checked this sentence for an explanation of the inconsistency.

It should be noted, however, that in Experiment 2 these eye movement patterns could be demonstrated only for readers who were able to report the inconsistency immediately after reading. For nonreporters - that is, readers who presumably did not notice the inconsistencyno such pattern was found. Instead, they tended to show an increase in their first-pass fixations of the critical target sentence, though it was numerically small and not statistically significant. Future research will have to determine whether this increase is equivalent to the signif- icant increase in sentence-reading times observed by Rinck et al. (2001). Although Experiment 2 was mainly designed to contrast noticed inconsistencies with unnoticed ones, it replicated the main result of the corresponding experiment by Rinck et al.: Even for nonreporters, the inconsistent version yielded longer total text fixation times than did the consistent one.

It should be noted that the distinction between reporters and nonreporters does not imply stability over time; that is, it should not be thought of as a personal trait. Instead, the very same person will probably notice some inconsistencies while being unaware of others. Moreover, the differences observed in Experiment 2 do not imply a perfect distinction between the two groups with regard to their reading behavior: Although regressions back to the second sentence differed between groups, the difference was far from perfect: About a third of the nonreporters regressed to the second sentence but still did not notice the inconsistency. Similarly, a third of the reporters did not regress to this sentence. Another problem with interpreting Experiment 2 is caused by the extremely limited set of materials. As a practical compromise, only two experimental texts could be used, so it was impossible to compute by-materials analyses. More research is needed to ensure that the results of Experiment 2 (and of Experiment 4 of Rinck et al., 2001) can be generalized to a larger set of materials. We are quite optimistic about this, however, because in general, our findings fit in well with earlier studies demonstrating the representation of temporal, spatial, emotional, goal-related, and character-related information, thereby confirming the multidimensional character of situation models postulated by recent theories of text comprehension (e.g., Zwaan, 1999; Zwaan, Langston, \& Graesser, 1995; Zwaan, Magliano, \& Graesser, 1995; Zwaan \& Radvansky, 1998). Most importantly, recent eye-tracking studies employing the inconsistency paradigm with expository texts (Lorch \& Lemberger, 2001) have yielded highly compatible results regarding eye movements.

From a methodological viewpoint, how do the eye movement patterns reported here relate to earlier results observed for sentence-by-sentence reading times? In general, they are quite compatible because both indicate that readers include temporal aspects in their situation models of the narratives, remain sensitive to temporal inconsistencies stated later in the texts, and show difficulties in integrating the inconsistent information into the situation model. Temporal order inconsistencies cause reliable increases in processing time both with sentencereading times and fixation times. Thus, the eye movement patterns observed here indicate that the inconsistency effect in target sentence-reading times is valid in the sense that it is indeed caused by the temporal inconsistency. They also indicate, however, that the effect may lack ecological validity: No increase in first-pass fixations of the target sentence was observed. If readers are able to see the complete text, they have a number of different op- 
tions for resolving an inconsistency: They may stay with the sentence containing the inconsistent information, they may go on reading in hope for clarification, or they may look back to related information. The sentence-bysentence presentation does not allow for the latter option: If readers search for related information, they have to perform a memory search instead of the visual search observed here. This may be advantageous from the researcher's point of view because it might increase the size of the inconsistency effect, but it is not necessarily representative of the processes occurring during everyday reading of text. In short, although the commonly used sentence-by-sentence reading paradigm reliably indicates that temporal inconsistencies cause some kind of additional processing, the eye movements reported here supply a better picture of what these additional processes are. Therefore, we suggest combining these approaches in future research on the construction, updating, and retrieval of situation models during text comprehension.

\section{REFERENCES}

Albrecht, J. E., \& O’Brien, E. J. (1993). Updating a mental model: Maintaining both local and global coherence. Journal of Experimental Psychology: Learning, Memory, \& Cognition, 19, 1061-1070.

Anderson, A., Garrod, S. C., \& Sanford, A. J. (1983). The accessibility of pronomial antecedents as a function of episode shifts in narrative text. Quarterly Journal of Experimental Psychology, 35A, 427-440.

Bestgen, Y., \& Vonk, W. (1995). The role of temporal segmentation markers in discourse processing. Discourse Processes, 19, 385-406.

Blanchard, H. E., \& Iran-NeJad, A. (1987). Comprehension processes and eye movement patterns in the reading of surprise-ending stories. Discourse Processes, 10, 127-138.

Carreiras, M., Carriedo, N., Alonso, M. A., \& Fernández, A. (1997). The role of verb tense and verb aspect in the foregrounding of information during reading. Memory \& Cognition, 25, 438-446.

COHEN, J. (1988). Statistical power analysis for the behavioral sciences. Hillsdale, NJ: Erlbaum.

DE VEGA, M. (1995). Backward updating of mental models during continuous reading of narratives. Journal of Experimental Psychology: Learning, Memory, \& Cognition, 21, 373-385.

DE Vega, M., Diaz, J. M., \& Leon, I. (1997). To know or not to know: Comprehending protagonists' beliefs and their emotional consequences. Discourse Processes, 23, 169-192.

GERNSBACHER, M. A. (1990). Language comprehension as structure building. Hillsdale, NJ: Erlbaum.

Gernsbacher, M. A., Goldsmith, H. H., \& Robertson, R. R. W. (1992). Do readers mentally represent characters' emotional states? Cognition \& Emotion, 6, 89-111.

Glenberg, A. M., \& Langston, W. E. (1992). Comprehension of illustrated text: Pictures help to build mental models. Journal of Memory \& Language, 31, 129-151.

Glenberg, A. M., Meyer, M., \& Lindem, K. (1987). Mental models contribute to foregrounding during text comprehension. Journal of Memory \& Language, 26, 69-83.

Graesser, A. C., Millis, K. K., \& ZwaAn, R. A. (1997). Discourse comprehension. Annual Review of Psychology, 48, 163-189.

HaengGi, D., Gernsbacher, M. A., \& Bolliger, C. A. (1994). Individual differences in situation-based inferencing during narrative text comprehension. In H. van Oostendorp \& R. A. Zwaan (Eds.), Naturalistic text comprehension (pp. 79-96). Norwood, NJ: Ablex.

Huitema, J. S., Dopkins, S., Klein, C. M., \& Myers, J. L. (1993). Connecting goals and actions during reading. Journal of Experimental Psychology: Learning, Memory, \& Cognition, 19, 1053-1060.

Johnson-LaIRD, P. N. (1983). Mental models. Cambridge: Cambridge University Press.
KaAkinen, J., Hyönä, J., \& Keenan, J. M. (2002). Individual differences in perspective effects on on-line text processing. Discourse Processes, 33, 159-173.

KINTSCH, W. (1988). The role of knowledge in discourse comprehension: A construction-integration model. Psychological Review, 95, 163-182.

Kintsch, W. (1998). Comprehension. New York: Cambridge University Press.

Lorch, R. F., JR., \& Lemberger, C. (2001, August). Detecting inconsistencies while reading. Poster presented at the Eleventh European Conference on Eye Movements, Turku, Finland.

MANDLER, J. M. (1986). On the comprehension of temporal order. Language \& Cognitive Processes, 1, 309-320.

MorRow, D. G. (1994). Spatial models created from text. In H. van Oostendorp \& R. A. Zwaan (Eds.), Naturalistic text comprehension (pp. 57-78). Norwood, NJ: Ablex.

Myers, J. L., Cook, A. E., Kambe, G., Mason, R. A., \& O'Brien, E. J. (2000). Semantic and episodic effects on bridging inferences. Discourse Processes, 29, 179-199.

O'Brien, E. J., \& Albrecht, J. E. (1992). Comprehension strategies in the development of a mental model. Journal of Experimental Psychology: Learning, Memory, \& Cognition, 18, 777-784.

Ohtsuka, K., \& Brewer, W. F. (1992). Discourse organization in the comprehension of temporal order in narratives. Discourse Processes, 15, 317-336.

RAYNER, K. (1998). Eye movements in reading and information processing: Twenty years of research. Psychological Bulletin, 124, 372422.

Rinck, M., HÄhnel, A., \& Becker, G. (2001). Using temporal information to construct, update, and retrieve situation models of narratives. Journal of Experimental Psychology: Learning, Memory, \& Cognition, 27, 67-80.

Schooler, J. W., \& Fiore, S. M. (1997). Consciousness and the limits of language: You can't always say what you think or think what you say. In J. D. Cohen \& J. W. Schooler (Eds.), Scientific approaches to consciousness (pp. 241-257). Mahwah, NJ: Erlbaum.

van DiJK, T. A., \& KinTSCH, W. (1983). Strategies of discourse comprehension. New York: Academic Press.

VAuras, M., HYÖNÄ, J., \& NiEMI, P. (1992). Comprehension of coherent and incoherent texts: Evidence from eye movement patterns and recall performance. Journal of Research in Reading, 15, 39-54.

Wiley, J., \& RAYNER, K. (2000). Effects of titles on the processing of text and lexically ambiguous words: Evidence from eye movements. Memory \& Cognition, 28, 1011-1021.

ZWAAN, R. A. (1996). Processing narrative time shifts. Journal of Experimental Psychology: Learning, Memory, \& Cognition, 22, 11961207.

ZWAAN, R. A. (1999). Five dimensions of narrative comprehension: The event-indexing model. In S. R. Goldman, A. C. Graesser, \& P. van den Broek (Eds.), Narrative comprehension, causality, and coherence: Essays in honor of Tom Trabasso (pp. 93-110). Mahwah, NJ: Erlbaum.

Zwaan, R. A., Langston, M. C., \& Graesser, A. C. (1995). The construction of situation models in narrative comprehension: An eventindexing model. Psychological Science, 6, 292-297.

ZwaAn, R. A., Madden, C. J., \& Whitten, S. N. (2000). The presence of an event in the narrated situation affects its availability to the comprehender. Memory \& Cognition, 28, 1022-1028.

Zwaan, R. A., Magliano, J. P., \& Graesser, A. C. (1995). Dimensions of situation model construction in narrative comprehension. Journal of Experimental Psychology: Learning, Memory, \& Cognition, 21, 386-397.

ZwaAn, R. A., \& Radvansky, G. A. (1998). Situation models in language comprehension and memory. Psychological Bulletin, 123, 162-185.

\section{NOTE}

1. In both experiments, the second-pass fixation durations also included durations of occasional third and fourth passes. These were rare, so they could not be analyzed separately. Moreover, analyses of secondpass fixations without these durations yielded the same effects. 


\section{APPENDIX \\ English Translation of Sample Text Used in Experiments 1 and 2}

1. It was a wonderful summer day.

2a. Consistent-Time Sentence:

In the morning, Alex had come to the coffee shop to meet Sabine, and he had an important interview scheduled for the afternoon.

2b. Inconsistent-Time Sentence:

In the morning, Alex had had an important interview, and in the afternoon, he came to the coffee shop to meet Sabine.

3. Alex and Sabine were journalists and longtime colleagues working at a large newspaper.

4. Today, they wanted to talk about a courtroom story that Sabine had been working on for weeks.

5. Alex was waiting for Sabine because she had received important news about the trial.

6. Target Sentence:

When Sabine finally entered the coffee shop, Alex was just about to leave for his important interview.

7. But his patience paid off because her news was good for a cover story.

Note-Each participant read either Sentence 2a or 2b. Texts were presented in Spanish in Experiment 1 and in German in Experiment 2.

(Manuscript received November 21, 2001;

revision accepted for publication August 23, 2002.) 